University of Texas Rio Grande Valley

ScholarWorks @ UTRGV

Chemistry Faculty Publications and

Presentations

College of Sciences

$12-1-2006$

\title{
Observation of Fano Asymmetry in Raman Spectra of SrTiO3 and Cax Sr1-xTiO3 Perovskite Nanocubes
}

\author{
Sarbajit Banerjee \\ Dae In Kim \\ Richard D. Robinson \\ Irving P. Herman \\ Yuanbing Mao \\ The University of Texas Rio Grande Valley, yuanbing.mao@utrgv.edu
}

See next page for additional authors

Follow this and additional works at: https://scholarworks.utrgv.edu/chem_fac

Part of the Chemistry Commons

\section{Recommended Citation}

Peterson, K. I., Pullman, D., Lin, W., Minei, A. J., \& Novick, S. E. (2007). Microwave spectra and ab initio studies of Ar-propane and Ne-propane complexes: Structure and dynamics. The Journal of Chemical Physics, 127(18), 184306. https://doi.org/10.1063/1.2780775

This Article is brought to you for free and open access by the College of Sciences at ScholarWorks @ UTRGV. It has been accepted for inclusion in Chemistry Faculty Publications and Presentations by an authorized administrator of ScholarWorks@ UTRGV. For more information, please contact justin.white@utrgv.edu,william.flores01@utrgv.edu. 


\section{Authors}

Sarbajit Banerjee, Dae In Kim, Richard D. Robinson, Irving P. Herman, Yuanbing Mao, and Stanislaus S. Wong

This article is available at ScholarWorks @ UTRGV: https://scholarworks.utrgv.edu/chem_fac/9 


\title{
Observation of Fano asymmetry in Raman spectra of $\mathrm{SrTiO}_{3}$ and $\mathrm{Ca}_{x} \mathrm{Sr}_{1-x} \mathrm{TiO}_{3}$ perovskite nanocubes
}

\author{
Sarbajit Banerjee, Dae-In Kim, Richard D. Robinson, and Irving P. Herman ${ }^{\text {a) }}$ \\ Materials Research Science and Engineering Center, Columbia University, New York, New York 10027 and \\ Department of Applied Physics and Applied Mathematics, Columbia University, New York, New York \\ 10027 \\ Yuanbing Mao \\ Department of Chemistry, Stony Brook University, Stony Brook, New York 11794
}

\author{
Stanislaus S. Wong \\ Department of Chemistry, Stony Brook University, Stony Brook, New York 11794 and Condensed Matter \\ Physics and Materials Science Department, Brookhaven National Laboratory, Upton, New York \\ 11973
}

(Received 7 August 2006; accepted 22 October 2006; published online 1 December 2006)

\begin{abstract}
Bulk $\mathrm{SrTiO}_{3}$ is cubic and not expected to exhibit any first-order Raman scattering. However, nanocubes of $\mathrm{SrTiO}_{3}$ with an edge length of $80 \pm 10 \mathrm{~nm}$ show strong first-order Raman scattering originating from the breaking of symmetry caused by frozen surface dipoles (local tetragonality) and the presence of nanoscopic polar domains (arising from incorporated impurities). Rapid polarization fluctuations within these nanoscopic ferroelectric regions interfere with a polar phonon, resulting in a Fano-like asymmetric line shape in these $\mathrm{SrTiO}_{3}$ nanocubes, as well as in $\mathrm{Ca}_{0.3} \mathrm{Sr}_{0.7} \mathrm{TiO}_{3}$ nanocubes. (C) 2006 American Institute of Physics. [DOI: 10.1063/1.2400095]
\end{abstract}

Ferroelectric perovskites, such as $\mathrm{SrTiO}_{3}$ and $\mathrm{Ca}_{x} \mathrm{Sr}_{1-x} \mathrm{TiO}_{3}$, have attracted much attention due to their nonlinear optical properties and electric-field dependent dielectric constants that make them potentially useful in phase and frequency agile electronics, monolithic microwave integrated circuits, and tunable high- $Q$ resonators. ${ }^{1}$ There has been interest in using thin films of ferroelectrics and incipient ferroelectrics (materials with unusual dielectric properties, such as suppressed paraelectric to ferroelectric transitions) to enable integration with microelectronics. ${ }^{2}$ However, the dielectric properties of incipient ferroelectrics can be quite different in thin film or nanostructured form as compared to bulk single crystals. ${ }^{3}$ While there have been advances in the controlled synthesis of nanocrystalline $\mathrm{SrTiO}_{3}$ and related perovskites, ${ }^{4-6}$ there has been relatively little progress in understanding how these systems differ from bulk $\mathrm{SrTiO}_{3}$. Such differences are observed here using Raman scattering. Raman spectroscopy can provide useful insight into the long- and short-range structures of these nanocrystalline materials and also into phonon absorption, which affects dielectric loss.

$\mathrm{SrTiO}_{3}$ nanocubes with an average edge length of $80 \pm 10 \mathrm{~nm}$, as determined by transmission electron microscopy (TEM) [Figs. 1(c) and 1(d)], are grown using a moltensalt solid-state reaction in the presence of $\mathrm{NaCl}^{4}$ The scanning electron microscopy (SEM) image in Fig. 1(a) shows clusters of these nanocubes. From x-ray diffraction (XRD) and selected area electron diffraction (SAED) patterns, these nanocrystals are cubic in structure $(P m 3 m)$ with a lattice constant $a=3.894 \AA$ [JCPDS value for cubic $\mathrm{SrTiO}_{3}$ of $a$ $=3.905 \AA$ (Ref. 7)]. ${ }^{5} \mathrm{CaTiO}_{3}$ nanostructures [Fig. 1(b)] and intermediate mixed perovskites with a composition of $\mathrm{Ca}_{x} \mathrm{Sr}_{1-x} \mathrm{TiO}_{3}$ are also synthesized by a modification of this molten salt technique. ${ }^{5}$ They are approximately nanocubes,

${ }^{a)}$ FAX: 1-212-854-1909; electronic mail: iph1@columbia.edu with lengths ranging from 70 to $110 \mathrm{~nm}$. XRD and $\mathrm{x}$-ray photoelectron spectroscopy measurements indicate the formation of pure phases without contamination from anatase or rutile titania. ${ }^{4-6}$ Moreover, TEM energy dispersive $\mathrm{x}$-ray and SAED observations do not show the presence of any $\mathrm{TiO}_{2}$ structures. We use Raman spectroscopy to study $\mathrm{SrTiO}_{3}$ and mixed perovskite $\mathrm{Ca}_{x} \mathrm{Sr}_{1-x} \mathrm{TiO}_{3}$ nanocubes using the $514.5 \mathrm{~nm}$ line of an Ar-ion laser, which are compared to bulk micrometer-sized $\mathrm{SrTiO}_{3}$ and $\mathrm{CaTiO}_{3}$ powder obtained from Alfa Aesar and Aldrich, respectively.

Bulk $\mathrm{SrTiO}_{3}$ has a centrosymmetric cubic structure at room temperature. Since all the zone-center optical phonons are of odd parity, no first-order Raman scattering is expected to occur. Instead broad second-order peaks are seen between 200 and $500 \mathrm{~cm}^{-1}$ and between 550 and $750 \mathrm{~cm}^{-1}$, as shown in Fig. 1(e). ${ }^{8}$ For $\mathrm{SrTiO}_{3}$ nanocubes, we observe clear firstorder peaks near $\sim 145,175$, and $545 \mathrm{~cm}^{-1}$ [Fig. 1(e)]. The first peak can be ascribed to the $E_{g}$ mode associated with noncentrosymmetric $\mathrm{SrTiO}_{3},{ }^{9}$, whereas the latter two modes originate from polar $\mathrm{TO}_{2}$ and $\mathrm{TO}_{4}$ phonons, which are associated with $\mathrm{O}-\mathrm{Ti}-\mathrm{O}$ bonding. ${ }^{10}$

First-order Raman scattering has been seen in $\mathrm{SrTiO}_{3}$ due to the breaking of symmetry by the application of an electric field, strain from lattice mismatch and grain boundaries, and doping by impurity atoms in the crystal lattice. ${ }^{10,11}$ For our freestanding $\mathrm{SrTiO}_{3}$ nanostructures, the lowering of symmetry likely arises from the presence of nanoscopic impurities incorporated in the lattice. The lowering of symmetry may also be associated with the frozen dipole moments at the nanocrystal surfaces. The polarization induced by these dipoles likely penetrates into the crystal, imparting a local tetragonal structure and destroying the inversion symmetry. ${ }^{12}$

A pronounced Fano asymmetric line shape is seen at the $\mathrm{TO}_{2}$ peak, which suggests coherent interference between the discrete phonon and a broad peak or continuum. ${ }^{13}$ Such asymmetric line shapes have been observed for polar $\mathrm{TO}_{2}$ 

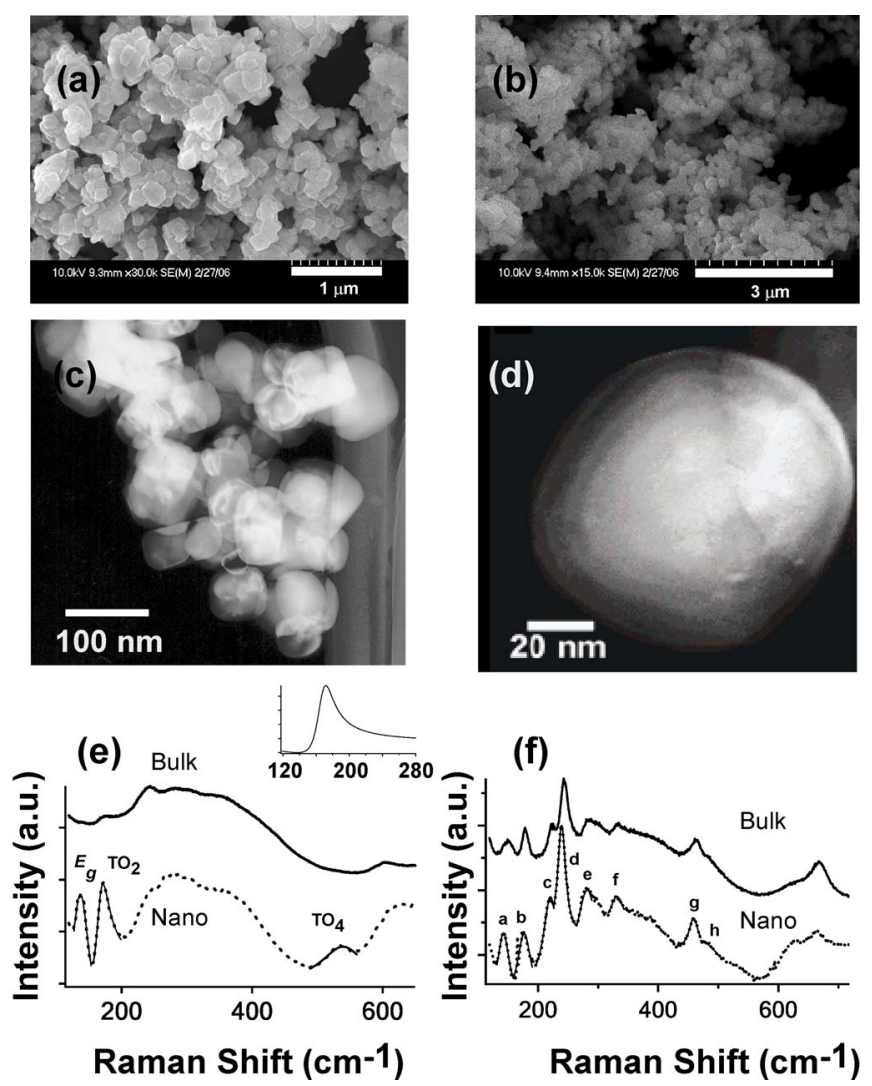

FIG. 1. (a) Scanning electron microscopy image of $\mathrm{SrTiO}_{3}$ nanocubes. SEM images were obtained on a Hitachi S4700 instrument with an accelerating voltage of $10 \mathrm{kV}$. (b) SEM image of $\mathrm{CaTiO}_{3}$ nanocubes. (c) TEM image of $\mathrm{SrTiO}_{3}$ nanocubes. (d) TEM image of a single nanocube. TEM images were obtained on a Philips CM12 instrument at an accelerating voltage of $120 \mathrm{kV}$. (e) Raman spectra of bulk $\mathrm{SrTiO}_{3}$ and $\mathrm{SrTiO}_{3}$ nanocubes at room temperature. The spectra for the nanocubes are represented by dashed lines. The two spectra are offset from each other for clarity. Fits to the $E_{g}, \mathrm{TO}_{2}$, and $\mathrm{TO}_{4}$ peaks for the $\mathrm{SrTiO}_{3}$ nanocubes are shown. $E_{g}$ and $\mathrm{TO}_{4}$ were fitted using Lorentzian line shapes, whereas the $\mathrm{TO}_{2}$ peak was fitted with a Fano profile (fits are superimposed on the spectra as solid lines). The Fano line shape is depicted more clearly in the inset to (e). The Fano fitting parameters are $A=0.38, q=5.07, \omega_{0}=168.4 \mathrm{~cm}^{-1}$, and $\Gamma=17.41 \mathrm{~cm}^{-1}$. (f) Raman spectra of bulk $\mathrm{CaTiO}_{3}$ and $\mathrm{CaTiO}_{3}$ nanocubes (dashed lines) at room temperature. All the peaks can be fitted with Lorentzian line shapes (shown for the nanocubes as solid lines). Peak $a$ is attributed to a $\mathrm{CaTiO}_{3}$ lattice mode, $b-f$ to the $\mathrm{O}-\mathrm{Ti}-\mathrm{O}$ bending modes, and $g$ to a torsional mode.

modes in $\mathrm{SrTiO}_{3}$ thin films and have been ascribed to the interaction of the phonon with a continuum arising from rapid polarization fluctuations in nanoscopic polar regions induced by impurities. ${ }^{10,14}$ Apparently, Fano line shapes have not been seen for nanocrystals previously.

The $E_{g}$ mode is not seen for epitaxially grown films and is likely observed in the nanocubes due to the frozen dipole moments at the surfaces, which break the inversion symmetry of the crystal; this is analogous to the effect of grain boundaries in $\mathrm{SrTiO}_{3}$ ceramics. ${ }^{12,15}$ Such a frozen dipole moment does not, however, explain the Fano line shape seen for the polar $\mathrm{TO}_{2}$ phonon. Since the phonons will not interact with static polarizations, there must be additional rapid polarization fluctuations, leading to the continuum response, which gives rise to the Fano lineshape. ${ }^{10}$ These rapid polarization fluctuations likely arise from the presence of defectinduced ferroelectric nanopolar regions in the nanoparticles. ${ }^{10}$ Defects could be present due to the incorporation of impurities or oxygen vacancies produced by insufficient oxygenation.
The $E_{g}$ and $\mathrm{TO}_{4}$ phonons in $\mathrm{SrTiO}_{3}$ nanocubes are fitted with Lorentzian line shapes, as shown in Figure 1(e). The Fano $\mathrm{TO}_{2}$ peak is fit by

$$
I(\omega)=A \frac{[q+E(\omega)]^{2}}{1+E(\omega)^{2}},
$$

after subtracting a constant base line, where

$$
E(\omega)=2\left(\omega-\omega_{0}\right) / \Gamma
$$

and $\omega_{0}$ is the phonon frequency, $A$ is the amplitude, $\Gamma$ is the full width at half maximum, and $q$ is the asymmetry parameter. This peak cannot be fitted with a Lorentzian plus a linear base line. The asymmetry parameter $q$ is always positive for these $\mathrm{SrTiO}_{3}$ nanocubes, which means that the scattering intensity is greater on the high-energy side of the peak. Interference with electronic continua is usually characterized by a negative value of $q$. Since the continuum interacts with a polar phonon, it is expected to be polar in nature, and thus may reasonably be ascribed to polarization fluctuations in defect-induced nanopolar regions. ${ }^{10}$ This would explain the absence of Fano broadening for the nonpolar $E_{g}$ mode which is not able to couple to the polar continuum. Since the $\mathrm{TO}_{4}$ peak is much higher in energy, it is thought that the density of states for the "continuum" vanishes at this frequency, and thus no Fano interference is seen for this mode, even though it has the same symmetry.

Phonon confinement is not significant for these nanocubes and does not explain any asymmetry in the Raman spectra, as is shown by simulations ${ }^{16}$ using the phonon dispersion curves for the $\mathrm{TO}_{2}$ phonon in $\mathrm{SrTiO}_{3} .{ }^{17}$ Using a "worst-case" scenario (maximum phonon dispersion in the model), the modeled spectra for $80 \pm 10 \mathrm{~nm}$ particles are the same as for bulk $\mathrm{SrTiO}_{3}$; finite size effects become significant only for particles $<5 \mathrm{~nm}$ in size.

Any clustering of the nanocubes, as seen in Fig. 1(a), will not affect the defects that lead to the Fano resonance. Such clustering would also not affect phonon confinement, as is clear from studies of nanocrystalline films.

Figure 2 shows the temperature dependence of the Fano peak. The asymmetry parameter $q$ increases and then decreases with temperature, as seen in Fig. 2(b), and this differs from the monotonic decrease for thin films in this temperature range. Not much is known about the precise behavior of the continuum of states arising from the dynamics of the local dipole moments in the local nanopolar regions. The asymmetry factor $q$ depends on the interaction strength between the phonon and the continuum states and on the density of the continuum states. ${ }^{18}$ A more systematic study as a function of size and impurity concentration is required to derive more definitive conclusions about the variation of the asymmetry with temperature.

Figure 1(f) compares the Raman spectra of bulk and nanoparticulate $\mathrm{CaTiO}_{3}$. The nanocubes are orthorhombic, just like the bulk, as confirmed by XRD and SAED observations. ${ }^{5}$ The first-order peaks for the $\mathrm{CaTiO}_{3}$ nanoparticles are superimposed on several broad second-order peaks and are fitted with Lorentzians and assigned to $\mathrm{CaTiO}_{3}$ lattice modes, O-Ti-O bending modes, and torsional modes. ${ }^{19}$

Figure 3 shows the room temperature Raman spectra for perovskites in the $\mathrm{SrTiO}_{3}-\mathrm{CaTiO}_{3}$ nanoparticle system. With increasing Sr content, most of the peaks broaden and shift to lower frequencies, as is observed for bulk samples. ${ }^{19,20}$ The peak position of the torsional $A_{g}$ mode is 


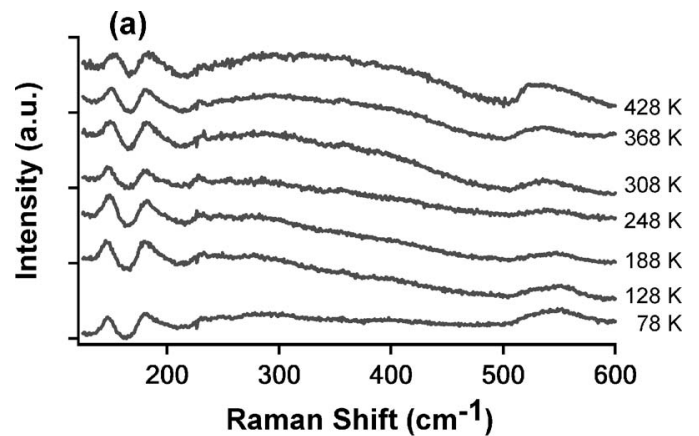

(b)

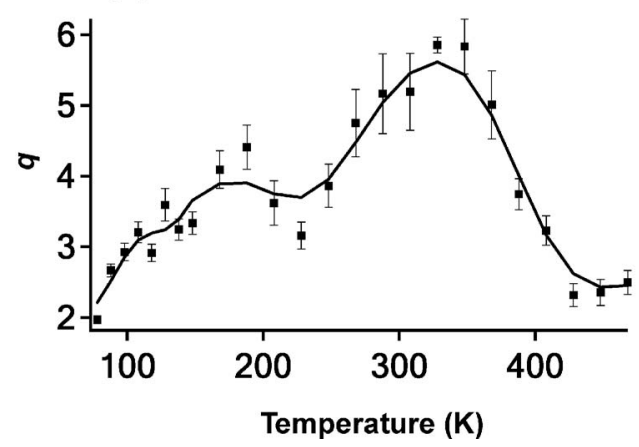

FIG. 2. (a) Representative Raman spectra obtained at different temperatures for $\mathrm{SrTiO}_{3}$ nanocubes. The temperature was controlled within $\pm 1{ }^{\circ} \mathrm{C}$ using a Linkam TMS 600 stage. (b) Temperature dependence of the asymmetry parameter $q$ as obtained by fitting the Fano profiles to Eq. (1).

plotted versus the $\mathrm{Sr}$ concentration in Fig. 3(b). The lower energy peaks are more dramatically affected by the increasing $\mathrm{Sr}$ concentration. A striking feature for the intermediate compositions $\mathrm{Ca}_{x} \mathrm{Sr}_{1-x} \mathrm{TiO}_{3}$ is the prominent $E_{g}$ peak, which

(a)
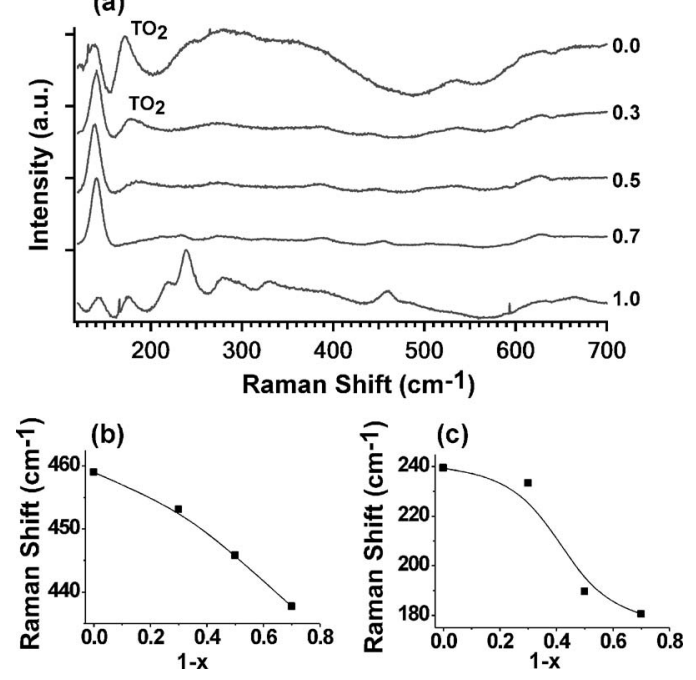

FIG. 3. (a) Room temperature Raman spectra for $\mathrm{Ca}_{x} \mathrm{Sr}_{1-x} \mathrm{TiO}_{3}$ nanocubes. [(b) and (c)] Plots depicting the peak position of structural modes identified in each with increasing $\mathrm{Sr}$ concentration. The $\mathrm{TO}_{2}$ mode is marked for the $x=0$ and 0.3 samples. is the highest intensity peak for all the intermediate compositions. In contrast, this peak is quite weak for bulk samples. ${ }^{19}$ This may again be due to the surface dipoles on the nanoparticles, which strongly favor the breaking of inversion symmetry. ${ }^{12,15}$ The $\mathrm{TO}_{2}$ phonon feature in $\mathrm{Ca}_{0.3} \mathrm{Sr}_{0.7} \mathrm{TiO}_{3}$ nanocubes displays a Fano asymmetry, but these features in more $\mathrm{Ca}$ rich nanocubes do not.

In summary, we present the first Raman measurements of $\mathrm{SrTiO}_{3}$ and $\mathrm{Ca}_{x} \mathrm{Sr}_{1-x} \mathrm{TiO}_{3}$ nanocrystals, which show behavior quite distinct from the bulk. Cubic $\mathrm{SrTiO}_{3}$ shows first-order Raman scattering due to permanent surface dipoles and the presence of nanoscopic polar regions, which break the inversion symmetry. The polar $\mathrm{TO}_{2}$ structural phonon feature in $\mathrm{SrTiO}_{3}$, and also $\mathrm{Ca}_{0.3} \mathrm{Sr}_{0.7} \mathrm{TiO}_{3}$, has an asymmetric Fano line shape attributed to the interference of the optical phonon with rapid polarization fluctuations in the nanopolar domains. Future work will focus on a more extensive delineation of the phase diagram for nanoparticulate $\mathrm{SrTiO}_{3}$.

This work was supported primarily by the MRSEC Program of the National Science Foundation under Award No. DMR-0213574 and the New York State Office of Science, Technology and Academic Research and partially by the NSEC Program of the National Science Foundation under Award No. CHE-0641523.

${ }^{1}$ J. F. Scott, Ferroelectr. Rev. 1, 1 (1998).

${ }^{2}$ R. Waser and D. M. Smyth, Ferroelectric Thin Films: Synthesis and Basic Properties (Gordon and Bneach, Amsterdam, 1996), Vol. 10, p. 47.

${ }^{3}$ R. Waser, Integr. Ferroelectr. 15, 39 (1997).

${ }^{4}$ Y. Mao, S. Banerjee, and S. S. Wong, J. Am. Chem. Soc. 125, 15718 (2003).

${ }^{5}$ Y. Mao and S. S. Wong, Adv. Mater. (Weinheim, Ger.) 17, 2194 (2005).

${ }^{6}$ Y. Mao, T.-J. Park, and S. S. Wong, Chem. Commun. (Cambridge) 2005, 5721.

${ }^{7}$ Joint Committee on Powder Diffraction Standards Card No. 73-0661 (International Center for Diffraction Data, Newton Square, PA).

${ }^{8}$ W. G. Nilsen and J. G. Skinner, J. Chem. Phys. 48, 2240 (1968).

${ }^{9}$ R. Ouillon, J.-P. Pinan-Lucarre, P. Ranson, P. Pruzan, S. K. Mishra, R. Ranjan, and D. Pandey, J. Phys.: Condens. Matter 14, 2079 (2002).

${ }^{10}$ A. A. Sirenko, I. A. Akimov, J. R. Fox, A. M. Clark, H.-C. Li, W. Si, and X. X. Xi, Phys. Rev. Lett. 82, 4500 (1999).

${ }^{11}$ J. M. Worlock and P. A. Fleury, Phys. Rev. Lett. 19, 1167 (1967).

${ }^{12}$ J. Petzelt and T. Ostapchuk, J. Optoelectron. Adv. Mater. 5, 725 (2003).

${ }^{13}$ U. Fano, Phys. Rev. 124, 1866 (1961).

${ }^{14}$ S. Gupta and R. S. Katiyar, J. Raman Spectrosc. 32, 885 (2001).

${ }^{15}$ J. Petzelt, T. Ostapchuk, I. Gregora, I. Rychetsky, S. Hoffmann-Eifert, A. V. Pronin, Y. Yuzyuk, B. P. Gorshunov, S. Kamba, V. Bovtun, J. Pokorny, M. Savinov, V. Porokhonskyy, D. Rafaja, P. Vanek, A. Almedia, M. R. Chaves, A. A. Volkov, M. Dressel, and R. Waser, Phys. Rev. B 64, 184111 (2001).

${ }^{16}$ J. E. Spanier, R. D. Robinson, F. Zhang, S.-W. Chan, and I. P. Herman, Phys. Rev. B 64, 245407 (2001).

${ }^{17}$ R. A. Cowley, Phys. Rev. 134, A981 (1964).

${ }^{18}$ P. H. M. van Loosdrecht, M. M. Maior, S. B. Molnar, Y. M. Vysochanskii, P. J. M. van Bentum, and H. van Kempen, Phys. Rev. B 48, 6014 (1993).

${ }^{19}$ S. Qin, X. Wu, F. Seifert, and A. I. Becerro, J. Chem. Soc. Dalton Trans. 2002, 3751.

${ }^{20}$ T. Hirata, K. Ishioka, and M. Kitajima, J. Solid State Chem. 124, 353 (1996). 